\title{
Prostatectomía radical robótica: revisión de nuestra curva de aprendizaje
}

\author{
Villavicencio Mavrich H, Esquena S, Palou Redorta J, Gómez Ruíz JJ.
}

Servicio de Urología. Fundació Puigvert. Barcelona.

Actas Urol Esp.2007; 31(6):587-592

\section{RESUMEN}

PROSTATECTOMIA RADICAL ROBÓTICA: REVISIÓN DE NUESTRA CURVA DE APRENDIZAJE

Objetivo: Presentamos los primeros casos de nuestra serie de prostatectomía radical robótica con Da Vinci (PRRdaV), que corresponden a la curva de aprendizaje (CA) del cirujano que se ha iniciado en la técnica.

Métodos: Se revisan los 20 primeros pacientes sometidos a PRRdaV y realizados por un cirujano experto, sin entrenamiento laparoscópico previo, pero con amplia experiencia en prostatectomía radical retropúbica y perineal (HV). Se analiza: tiempo operatorio, pérdidas hemáticas, tasa de conversión, complicaciones intra y postoperatorias, estancia hospitalaria y días de sonda vesical. También: las tasas y la localización de los márgenes positivos, así como los resultados funcionales con un seguimiento medio de 10 meses.

Resultados: La media de tiempo operatorio fue de 140 minutos (100-211) y la pérdida hemática media de $180 \mathrm{~mL}(80-$ 360), no requiriendo transfusión sanguínea en ningún caso. No se presentaron complicaciones intraoperatorias, y tampoco ninguna reconversión. Como acontecimientos postoperatorios sólo destaca una retención aguda urinaria tras retirada de sonda vesical. La estancia hospitalaria media fue 3,35 días (3-5). Se obtuvieron 6 casos con márgenes quirúrgicos positivos (30\%). La localización más frecuente fue postero-lateral. Dieciocho de los 20 pacientes (90\%) son completamente continentes de forma precoz, $2(10 \%)$ requirieron utilizar una compresa de seguridad durante los 6 primeros meses por incontinencia leve de esfuerzo que se resolvió espontáneamente. De los 20 casos, 2 de ellos (10\%) presentaban disfunción eréctil preoperatoria; de los 18 restantes, 12 (66.6\%) conservaban potencia en el momento de la revisión y 6 (33.4\%) presentaron disfunción eréctil postoperatoria.

Conclusiones: La prostatectomía radical mediante cirugia robótica se ha demostrado claramente ventajosa (excelente maniobrabilidad para una técnica minimamente invasiva, postura más cómoda y anatómica, visión tridimensional y corta curva de aprendizaje). Una amplia experiencia quirúrgica previa en cirugía abierta y/o laparoscópica, acortan de forma significativa esta curva de aprendizaje de la PRRdaV.

Palabras clave: Da Vinci. Curva de aprendizaje. Cáncer de próstata. Prostatectomía radical robótica.

\section{ABSTRACT}

ROBOTIC RADICAL PROSTATECTOMY: OVERVIEW OF OUR LEARNING CURVE

Objective: We present the first cases of our robotic radical prostatectomy with Da Vinci (RRPdaV) that corresponds to the learning curve $(\mathrm{LC})$ of the surgeon that has initiated with this technique.

Methods: We reviewed the first 20 patients that underwent RRPdaV, performed by an expert surgeon, without previous laparoscopic training, but with a wide experience in retropubic and perineal prostatectomy (HV). We analyzed: Surgical time, blood loss, conversion rate, intra and postoperative complications, hospital stay and days of bladder catheterization. Also: rates and location of surgical margins, as well as functional outcomes with an average follow up of 10 months. Results: Mean operating time was 140 minutes (100-211) and blood loss $180 \mathrm{~mL}$ (80-360), and none required a blood transfusion. There were no intraoperative complications and neither any conversion to open surgery. The only postoperative outstanding fact was mean hospital stay were 3,35 days. (3-5). We had 6 cases of positive surgical margins (30\%). The most frequent location was postero-lateral. Eighteen out of 20 patients (90\%) were early totally continent, 2 (10\%) required the use of one pad during the first six months due slight stress incontinence that stopped spontaneously. From 20 cases, two of them (10\%) had preoperative erectile dysfunction; 12 out of the remaining $18(66.6 \%)$ preserved potency at review and $6(33.4 \%)$ had postoperative erectile dysfunction.

Conclusions: It has been demonstrated that robotic surgery for radical prostatectomy is clearly an advantage technique (easy maneuver although it is a minimally invasive technique, comfortable and ergonomic position for the surgeon, 3D visualization and short learning curve). The RRPDAv learning curve is significantly shorter if the surgeon has a wide previous surgical experience in open and/or laparoscopic surgery.

Keywords: Da Vinci. Learning curve. Prostate cancer. Robotic radical prostatectomy. 
$\mathrm{L}^{2}$ a cirugía robótica mediante el sistema Da Vinci (Intuitive Surgical, Sunnyvale, CA, EE.UU) ha situado nuevamente a la Urología a la cabeza de la cirugía mínimamente invasiva. El número de actos quirúrgicos urológicos realizados en el mundo con este robot, ha pasado de 1.500 en el año 2000 a 20.000 en el año $2005^{1}$ y 36.600 en el 2006. Aunque en la actualidad la indicación principal es la prostatectomía radical, su uso se está ampliando rápidamente a otras indicaciones urológicas (nefrectomía radical y parcial, pieloplastia, cistectomía, linfadenectomía retroperitoneal) y así hasta un total de 22 procedimientos.

Desde que en Europa, el Dr. Richard Gaston realizara en septiembre de 1997 la primera prostatectomía radical laparoscópica (PRL) y hasta la actualidad, este procedimiento se está convirtiendo en la técnica estándar para el tratamiento quirúrgico del cáncer de próstata localizado. La morbilidad perioperatoria, los requerimientos analgésicos, el tiempo de recuperación y las pérdidas sanguíneas de la cirugía abierta están provocando el abandono de esta técnica por la laparoscópica. La implantación de la PRL se ha encontrado con una larga y compleja curva de aprendizaje (CA), que a pesar de las ventajas de la técnica, está limitando su generalización en nuestro medio. La cirugía robótica mediante el sistema Da Vinci incorpora las ventajas de un abordaje laparoscópico junto con las de una visión tridimensional, una mayor maniobrabilidad y precisión, así como una gran comodidad para el cirujano, ventajas propias e incluso mejores a la cirugía abierta, sumadas a una gran reducción de la CA.

Series norteamericanas y europeas abogan porque la prostatectomía radical robótica con Da Vinci (PRRdaV) se constituya y sustituya a la PRL como la técnica de elección para el tratamiento quirúrgico del cáncer de próstata. El coste actual del robot, de aproximadamente un millón y medio de euros, y el gasto de unos 800-1.200 euros de material fungible por cada procedimiento, no permiten esperar la misma generalización en nuestro país debido a la particular estructura y financiación del sistema sanitario español.

La primera PRRdaV se realizó en Frankfurt en Mayo de 2000, por el Dr. J. Binder, un urólogo con amplia experiencia en cirugía abierta pero sin ninguna experiencia laparoscópica ${ }^{2}$. En Estados Unidos, la primera intervención se realizó en noviembre de 2000, en el Vattikuti Institute de Urologia de Detroit por el Dr. G. Vallencien, durante un programa de adiestramiento ${ }^{3}$. Posteriormente el equipo de este Instituto, liderado por el Dr. M. Menon, describió su técnica original realizando más de 1.000 procedimientos hasta $2004^{4,5}$. La primera PRRdaV en España se realizó en julio de 2005 en la Fundació Puigvert de Barcelona, por el Dr. H. Villavicencio ${ }^{6}$ que, al igual que Binder y Menon, no contaba con experiencia laparoscópica previa.

Por otro lado, el impacto positivo de la robótica, cada vez es más imparable, así por ejemplo, en EE.UU, en el 2005 (Fig. 1), el 20\% de las prostatectomías se realizaron con el robot Da Vinci, el $0.7 \%$ con laparoscopia y el 79,3\% por vía abierta. En el 2006 (Fig. 2), supuso el 41,5\% con el robot, el $0,5 \%$ con laparoscopia y el $58,5 \%$ por vía abierta, y la proyectada para el 2007 (Fig. 3) ya representará una superioridad para la robótica con el 63,0\%, siendo el 0,2\% para la laparoscopia y el $36,85 \%$ para la vía abierta (IntuitiveSurgical.com).

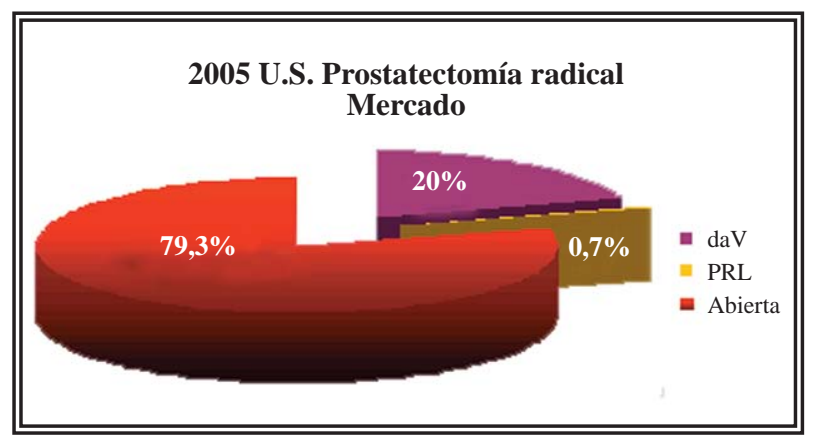

FIGURA 1

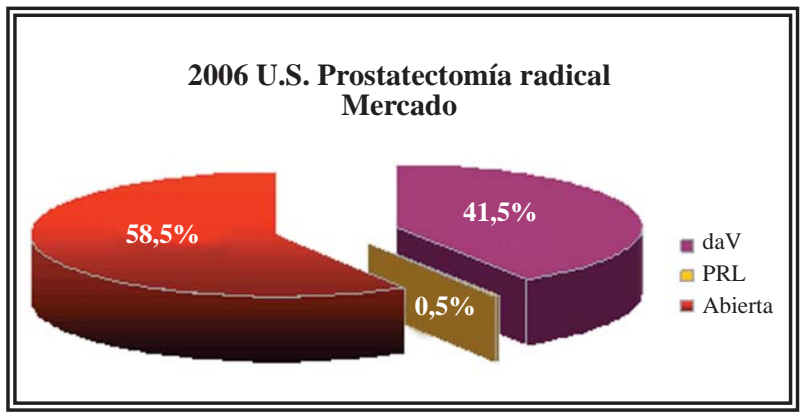

FIGURA 2 


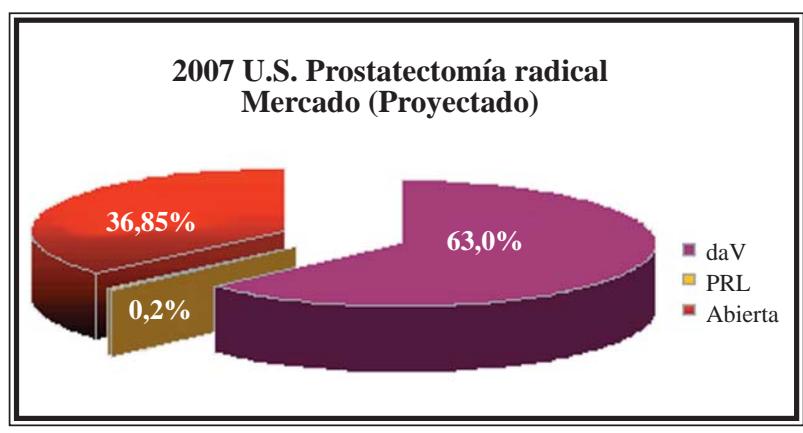

FIGURA 3

\section{MATERIAL Y MÉTODOS}

Entre julio de 2005 y noviembre de 2005, se realizaron en nuestra Institución las primeras 20 PRRdaV (cirugía asistida por robot) en pacientes con cáncer de próstata localizado. Todos los procedimientos fueron ejecutados por el mismo cirujano (H. Villavicencio) En todos los casos la intervención se realizó mediante un acceso transperitoneal. Aunque en la actualidad la Institución cuenta con más de 100 procedimientos, sólo se revisan los 20 primeros, correspondientes a la CA definida por el propio cirujano, después de la experiencia acumulada hasta hoy. La edad media de los pacientes fue de 61 años (46-70). El peso medio de la glándula determinado por ecografía transrectal fue 34,6 gramos (17-49). La media de PSA preoperatorio fue $7,4 \mathrm{ng} / \mathrm{mL}(4,59-17,7)$. El estadio clínico fue Tlc en 18 pacientes, Tlb en otro, obtenido después de una RTU de próstata, y un T2a. Se analizaron el tiempo operatorio, las pérdidas hemáticas, la tasa de conversión, las complicaciones intra y postoperatorias, la estancia hospitalaria y los días de sonda vesical. También se revisaron las tasas de márgenes positivos así como su localización. Con un seguimiento medio de 10 meses se evalúan los resultados funcionales.

\section{RESULTADOS}

Definimos el tiempo operatorio desde que el cirujano se coloca en la consola del robot hasta que se retira de la misma. Este tiempo no comprende la colocación del robot por un equipo de enfermería entrenado y la colocación trócares y brazos del robot, que requiere unos 40 minutos, destacando que el proceso se reduce de forma considerable a medida que el equipo auxiliar gana experiencia. La media de tiempo operatorio fue de 140 minutos (100-211), es decir, 2 horas (h) y 20 minutos (min) (3h 31min-1h $40 \mathrm{~min}$ ), destacando que la duración del primer procedimiento de la serie fue $3 \mathrm{~h} 30$ min y la del último, 1 h 40 min (Fig. 4). La pérdida hemática media fue de $180 \mathrm{ml}$ (80-360), no requiriendo transfusión sanguínea en ningún caso. No hubo complicaciones intraoperatorias, y tampoco ninguna reconversión. Como acontecimientos postoperatorios sólo destaca una retención aguda urinaria tras retirada de sonda vesical, que únicamente obligó a recolocarla durante una semana más. La estancia hospitalaria media fue 3,35 días (2-5). Se retiró la sonda vesical a todos los pacientes el día 14 después de la intervención, realizando cistografía de relleno previa de control por tratarse de los primeros casos. Todos los pacientes reanudaron micciones espontáneas, excepto en el caso descrito previamente de la retención.

En relación a los resultados oncológicos, y después del análisis anatomo-patológico de las piezas quirúrgicas, se obtuvieron 6 casos con márgenes quirúrgicos positivos, representando una 30\% del total. De éstos, el 50\% eran irregulares. La localización más frecuente fue posterolateral (4 casos), 1 caso en ápex y otro en región

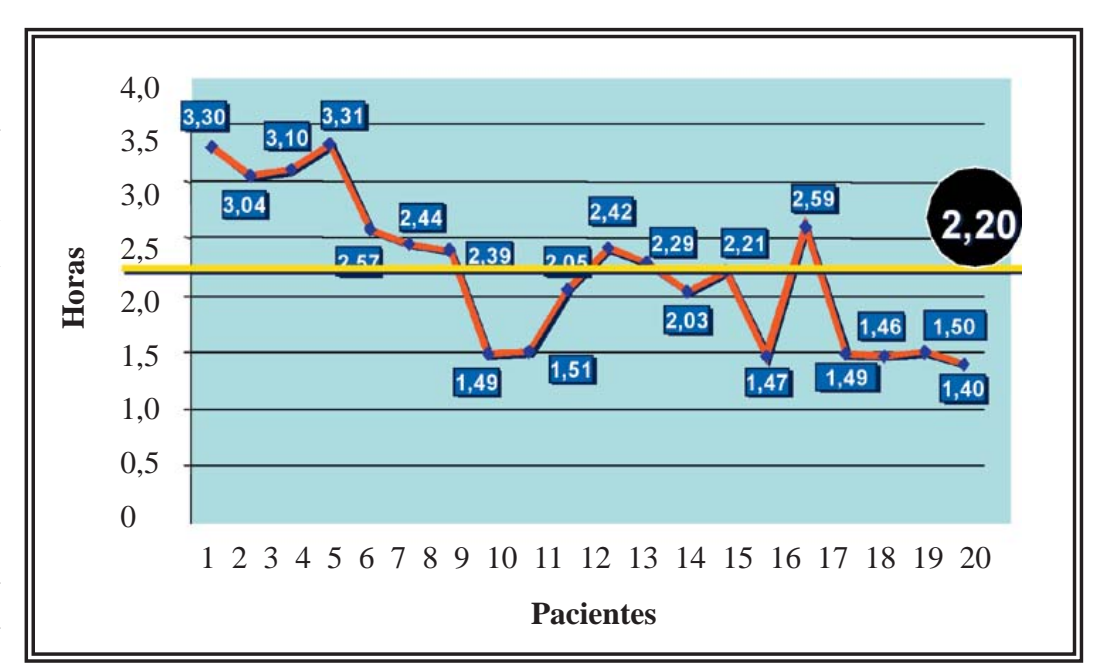

FIGURA 4. Tiempo operatorio medio y detallado de cada PRRdaV durante los 20 primeros casos de la curva de aprendizaje del Dr. Villavicencio en la Fundació Puigvert. 
fibromuscular anterior. En cuanto al estadio patológico, 11 casos (55\%) fueron pT2c, 6 casos (30\%) pT2a, y los restantes 3 casos fueron pT0 (T1b post-RTU), pT2b y pT3b, representando un $5 \%$ cada uno (Tabla 1). Hasta el día de hoy, todos los pacientes presentan un PSA indetectable.

\section{Tabla 1}

Relación de estadios patológicos y márgenes quirúrgicos de nuestra serie.

\begin{tabular}{lrrrr}
\hline \multicolumn{2}{l}{ Estadio patológico } & \multicolumn{2}{c}{ Márgenes quirúrgicos } \\
\hline pT0 & 1 & $5 \%$ & & - \\
pT2a & 6 & $30 \%$ & 1 & $5 \%$ \\
pT2b & 1 & $5 \%$ & & - \\
pT2c & 11 & $55 \%$ & 4 & $20 \%$ \\
pT3b & 1 & $5 \%$ & 1 & $5 \%$ \\
TOTAL & 20 & $100 \%$ & 6 & $30 \%$ \\
\hline
\end{tabular}

En relación a los resultados funcionales, $18 \mathrm{de}$ los 20 pacientes (90\%) son completamente continentes y de forma precoz, no habiendo requerido del uso de compresas en ningún momento. Dos pacientes (10\%) presentan urgencia miccional, de los que uno de ellos padece enfermedad de Parkinson. Además, éstos requirieron utilizar una compresa de seguridad durante los 6 primeros meses por incontinencia leve de esfuerzo que se resolvió espontáneamente. En cuanto a la función sexual, de los 20 pacientes evaluados, 2 de ellos (10\%) presentaban disfunción eréctil preoperatoria. De los 18 restantes, 12 (66,6\%) conservaban potencia en el momento de la revisión, y $6(33,4 \%)$ pacientes presentaron disfunción eréctil postoperatoria (tiempo de seguimiento medio 10 meses (8-12).

\section{DISCUSIÓN}

En los últimos años, la implantación y desarrollo progresivo de técnicas quirúrgicas mínimamente invasivas, como la laparoscopia y la robótica, han popularizado el concepto de CA, cuya definición es el número de casos que un cirujano debe realizar para conseguir la suficiencia o maestría en una técnica quirúrgica con el mínimo de complicaciones ${ }^{7}$. No está descrito un estándar definido de casos, pero sería el número a partir del cual el cirujano se siente cómodo realizando el procedimiento. La CA puede variar sustancialmente obedeciendo a múltiples factores dependientes del cirujano, destacando entre ellos una amplia experiencia quirúrgica previa. Un hecho que parece extremadamente evidente es que la CA de la PRL es larga y complicada, mientras que en la PRRdaV es todo lo contrario, ya que el uso del robot facilita tanto la sutura como otros aspectos que se hallan entre los más complejos del aprendizaje laparoscópico, cuestión fundamental en un futuro para conseguir una generalización de la técnica. Así como algunos trabajos informan que para obtener la suficiencia necesaria para realizar la PRL en 4 horas son necesarios entre 40 y $60 \operatorname{casos}^{8,9}$, otros autores muestran que, sin experiencia laparoscópica previa, a partir de 10 casos se puede obtener esta suficiencia para realizar una PRRdaV en 4 horas $^{10,11}$. La experiencia de nuestra serie confirma esos resultados e incluso los mejora en tiempo quirúrgico ya que, a partir del caso número 10 , la mayoría de procedimientos duraron menos de 2 horas.

Independientemente de los factores relacionados con el cirujano, el acortamiento de la CA es debido sobre todo, a las condiciones de trabajo quirúrgico ofrecidas por el robot. Los brazos de un ser humano tienen 29 grados de libertad de movimiento realizados en 3 ejes, posibilitando la realización de 594.823.321 movimientos. El robot Da Vinci tiene 7 grados, capacitándole para realizar 117.649 movimientos $(0,019 \%$ del total de la capacidad del brazo humano), cercanos a los que utiliza en realidad un cirujano en una intervención convencional. Esta cifra es muy superior a los 3 grados de libertad y 729 movimientos posibles en cirugía laparoscópica. Por otro lado, el efecto "fulcrum" de la laparoscopia consistente en que el cirujano debe realizar los movimientos en la dirección opuesta a los que ve en la pantalla para llegar al punto de profundidad deseado, es superado por Da Vinci, ofreciendo al cirujano una percepción real de la dirección y la profundidad en que se mueven los instrumentos, así como un movimiento muy intuitivo de los dedos, que junto con la confortable posición del cirujano permiten una óptima visualización y precisión que facilita la realización de todos los movimientos necesarios para 
la ejecución de la cirugía. Cabe destacar que el único inconveniente de la robótica es la pérdida de la sensación táctil a la que está habituado el cirujano, que queda ampliamente suplida por las ventajas descritas anteriormente.

La visión tridimensional de alta resolución junto con las grandes posibilidades de movimiento del robot en sus 7 grados de libertad, permiten a un cirujano con escasa o nula experiencia laparoscópica, acortar significativamente su CA. Aunque ya hemos comprobado que la literatura todavía no permite definir un número de casos de CA, 20 procedimientos parece un límite razonable para realizar un PRRdaV con un tiempo operatorio y una tasa de complicaciones aceptables $^{3,10,12,13}$.

$\mathrm{Si}$ revisamos los datos de las curvas de aprendizaje de PRRdaV de otros grupos (Tabla $2)^{14}$, es importante destacar que la media de pacientes incluidos en estas series iniciales es de 40. Además observamos que los tiempos quirúrgicos se reducen progresivamente a medida que aumenta la experiencia del cirujano. Bentas et al. ${ }^{13}$ reporta un descenso sucesivo de 22 minutos por cada procedimiento realizado, aunque también reporta la mayor tasa de complicaciones $(41,7 \%)$, siendo la fuga anastomótica la más frecuente y presenta la mayor tasa de transfusión (32\%). Por otro lado, Ahlering et al. ${ }^{10}$ obtiene una suficiencia de 4 horas a partir de los 12 casos realizados, con unos datos paralelos a los obtenidos en nuestra serie. Además, señala que los pasos en los que se rebaja más tiempo a largo de la CA son la disección del cuello y vesículas seminales, y la anastomosis vesico-uretral. En todas las series la tasa de conversión no superó el 5\%. En cuanto a los resultados oncológicos, las tasas de márgenes positivos son muy variables, desde un $2 \%^{15}$ a un $59 \%{ }^{16}$, estableciéndose una clara relación directa con el estadio patológico, y como se constata en el seguimiento, disminuyendo de forma progresiva con la experiencia del cirujano. En relación a los resultados funcionales, el seguimiento a 12 meses muestra una tasas de continencia entre el $84 \%$ y el $98 \% 13,17$ y en los pacientes a los que se realizó preservación de bandeletas, entre un $20 \%$ y un $97 \%$ mantenían potencia sexual después de la intervención ${ }^{13,15}$.

Es de gran importancia destacar que para obtener estos resultados el cirujano debe disponer de un ayudante con experiencia en laparoscopia, que permanece junto al paciente. El personal auxiliar debe estar involucrado de forma regular en cirugía robótica con objeto de cuidar detalles como la correcta colocación inicial del paciente y la instrumentación propia de la cirugía con Da Vinci.

$\mathrm{Si}$ el cirujano posee experiencia quirúrgica laparoscópica previa, la CA podría ser todavía más reducida debido a la afinidad entre ambas técnicas.

Lo mismo se podría extrapolar a un cirujano con experiencia en cirugía abierta y conocedor de

\section{Tabla 2}

Experiencias iniciales reportadas en PRRdaV de más de 10 casos, extraídas de Ficarra et al. ${ }^{14}$, y completada con nuestra serie. Nr: No reportado en la serie.

\begin{tabular}{|c|c|c|c|c|c|c|c|c|c|}
\hline Autor & Año & $\begin{array}{c}\text { № } \\
\text { casos }\end{array}$ & $\begin{array}{c}\text { Tiempo } \\
\text { operatorio } \\
\text { (min) }\end{array}$ & $\begin{array}{c}\text { Pérdida } \\
\text { hemática } \\
(\mathrm{mL})\end{array}$ & $\begin{array}{c}\text { Tasa } \\
\text { transfusión } \\
\text { (\%) }\end{array}$ & $\begin{array}{c}\text { Tasa } \\
\text { conversión } \\
(\%)\end{array}$ & $\begin{array}{c}\text { Tasa } \\
\text { complicaciones } \\
\text { (\%) }\end{array}$ & $\begin{array}{c}\text { Estancia } \\
\text { hospitalaria } \\
\text { (dias) }\end{array}$ & $\begin{array}{l}\text { Retirada } \\
\text { sonda } \\
\text { (dias) }\end{array}$ \\
\hline Menon & 2002 & 40 & 274 & 256 & $0 \%$ & $0 \%$ & $5 \%$ & 1 & $\mathrm{Nr}$ \\
\hline Menon & 2002 & 30 & 288 & 329 & $7 \%$ & $3 \%$ & $20 \%$ & 1.5 & 10,7 \\
\hline Bentas & 2003 & 41 & 498 & 570 & $32 \%$ & $5 \%$ & $41.7 \%$ & 17 & 16,7 \\
\hline Ahlering & 2003 & 45 & 225 & 145 & $0 \%$ & $0 \%$ & $8.8 \%$ & 1.5 & 7 \\
\hline Sim & 2004 & 17 & 247 & 494 & $18 \%$ & $0 \%$ & $6 \%$ & 2,7 & 9,8 \\
\hline Chien & 2005 & 56 & 354 & 356 & $2 \%$ & $0 \%$ & $9 \%$ & 1.9 & 6,6 \\
\hline Patel & 2005 & 50 & 202 & 151 & $0 \%$ & $0 \%$ & $1 \%$ & $\mathrm{Nr}$ & $\mathrm{Nr}$ \\
\hline Villavicencio & 2007 & 20 & 140 & 180 & $0 \%$ & $0 \%$ & $5 \%$ & 3,3 & 14 \\
\hline
\end{tabular}


la robótica, en el que también sería más sencillo su aprendizaje laparoscópico. En general, es recomendable pero no imprescindible, que un cirujano abierto experto, realice un entrenamiento previo en laparoscopia como preparación a la cirugía robótica, tanto para reducir su CA como para desarrollar habilidad quirúrgica en un campo laparoscópico, que permitirá solucionar por esta misma vía las posibles complicaciones postoperatorias de una cirugía robótica.

Por otro lado, como ha sucedido en otras revoluciones de alta tecnología, a medida que avanza su difusión se abaratan progresivamente los costes, hecho que podría comportar una generalización en nuestro país de la robótica, pero de forma mucho más tardía respecto al resto a países europeos y de Estados Unidos, hecho que alejaría a nuestro sistema sanitario del máximo nivel médico y tecnológico.

\section{CONCLUSIONES}

La realización de la prostatectomía radical mediante cirugía robótica se ha demostrado claramente ventajosa (excelente maniobrabilidad para una técnica compleja y mínimamente invasiva, con mejor ergonomía, visión tridimensional, y gran precisión). Además está demostrando resultados absolutamente competitivos y mejorados a nivel oncológico y funcional en relación a otras técnicas, destacando especialmente por su corta curva de aprendizaje.

$\mathrm{El}$ sistema quirúrgico robotizado Da Vinci, ofrece a los cirujanos una herramienta sofisticada que permite realizar suturas difíciles en delicadas y complicadas intervenciones.

Una amplia experiencia quirúrgica previa en cirugía abierta, acorta de forma significativa esta curva de aprendizaje de la PRRdaV que, en nuestra experiencia, se sitúa sobre los 20 casos.

\section{REFERENCIAS}

1. Carol Marie Cropper. Business Week, March 14, 2005.

2. Binder J, Kramer W. Robotically-assisted laparoscopic radical prostatectomy. BJU Int. 2001 Mar;87(4):408-410.

3. Menon M, Shrivastava A, Tewari A, Sarle R, Hemal A, Peabody JO, Vallancien G. Laparoscopic and robot assisted radical prostatectomy: establishment of a structured program and preliminary analysis of outcomes. J Urol. 2002 Sep; 168(3):945-949.
4. Tewari A, Peabody J, Sarle R, Balakrishnan G, Hemal A, Shrivastava A, Menon M. Technique of da Vinci robotassisted anatomic radical prostatectomy. Urology 2002 Oct;60 (4):569-572.

5. Menon M, Tewari A, Peabody JO, Shrivastava A, Kaul S, Bhandari A, Hemal AK. Vattikuti Institute prostatectomy, a technique of robotic radical prostatectomy for management of localized carcinoma of the prostate: experience of over 1100 cases. Urol Clin North Am. 2004 Nov;31(4):701717.

6. Villavicencio Mavrich H. Cirugía laparoscópica avanzada robótica Da Vinci: origen, aplicación clínica actual en Urología y su comparación con la cirugía abierta y laparoscópica. Actas Urol Esp. 2006 ;30(1):1-12.

7. Grillo HC. To impart this art: The development of graduate surgical education in the United States. Surgery 1999; 125(1): 1-14.

8. Guillonneau B, Rozet F, Barret E, Cathelineau X, Vallancien G. Laparoscopic radical prostatectomy: assessment after 240 procedures. Urol Clin North Am. 2001 Feb; 28(1): 189-202.

9. Kavoussi LR. Laparoscopic radical prostatectomy: irrational exuberance?. Urology. 2001 Oct; 58(4):503-505.

10. Ahlering TE, Skarecky D, Lee D, Clayman RV. Successful transfer of open surgical skills to a laparoscopic environment using a robotic interface: initial experience with laparoscopic radical prostatectomy. J Urol. 2003 Nov; 170(5): 1738-1741.

11. Meadows M. Robots lend a helping hand to surgeons. FDA Consumer2002 May-Jun;36(3):10-15.

12. Menon M, Tewari A, Baize B, Guillonneau B, Vallancien G. Prospective comparison of radical retropubic prostatectomy and robot-assisted anatomic prostatectomy: the Vattikuti Urology Institute experience. Urology. 2002 Nov; 60(5):864-868,.

13. Bentas W, Wolfram M, Jones J, Brautigam R, Kramer W, Binder J. Robotic technology and the translation of open radical prostatectomy to laparoscopy: the early Frankfurt experience with robotic radical prostatectomy and one year follow-up. Eur Urol. 2003 Aug;44(2):175-181.

14. Ficarra V, Cavalleri S, Novara G, Aragona M, Artibani W. Evidence from robot-assisted laproscopic radical prostatectomy: a systematic review . Eur Urol. 2007; 51(1):45-55.

15. Menon M, Kaul S, Bhandari A, Shrivastava A, Tewari A, Hemal A. Potency following robotic radical prostatectomy: a questionnaire based analysis of outcomes after conventional nerve sparing and prostatic fascia sparing techniques. J Urol. 2005 Dec; 174(6):2291-2296.

16. Sim HG, Yip SK, Lau WK, Tan JK, Cheng CW. Early experience with robot-assisted laparoscopic radical prostatectomy. Asian J Surg. 2004 Oct;27(4):321-325.

17. Patel VR, Tully AS, Holmes R, Lindsay J. Robotic radical prostatectomy in the community setting-the learning curve and beyond: initial 200 cases. J Urol. 2005 Jul;174 (1):269-272.

Correspondencia autor: Dr. H. Villavicencio Mavrich Servicio de Urología. Fundació Puigvert

Cartagena, 340-350 - 08025 Barcelona. Tel.: 934169700

E-mail autor: hvillavicencio@fundacio-puigvert.es

Información artículo: Original 